\title{
Post-Acute Sequelae of SARS-COVID-2 Syndrome: Just the Beginning
}

\author{
Shereif H. Rezkalla, b, e, f, Robert A. Kloner ${ }^{\mathrm{c}, \mathrm{d}, \mathrm{e}}$
}

\begin{abstract}
Viral diseases are some of the most common infections affecting humans. Despite the unpleasant symptoms, most people return to their normal lives without residual symptoms. Following the acute infectious phase of some viruses, however, in some individuals symptoms may linger to the extent they are unable to return to a normal lifestyle. Following coronavirus disease 2019 infection, significant numbers of patients continued to have symptoms that persisted for months after hospital discharge. Symptoms spanned many organ systems and were prominent in the pulmonary and cardiovascular systems. The exact mechanism is not clear. This group of patients represents a new challenge to our health care systems. An organized, multi-disciplinary approach and further research are warranted to be ready to deliver better care to these patients.
\end{abstract}

Keywords: Post-acute; SARS-COVID-2; PASC; Long haulers; Multi-systemic

\section{Introduction}

Viral diseases are some of the most common infections affecting human beings. According to the National Center of Health Statistics, the common cold virus causes 35.6 infections per 100 persons annually [1]. Despite the unpleasant symptoms, most people return to normal and are back to the workforce without residual symptoms. Following the acute infectious phase

Manuscript submitted June 10, 2021, accepted June 24, 2021

Published online August 31, 2021

aDepartment of Cardiology \& Cardiovascular Research, Marshfield Clinic Health System, 1000 North Oak Avenue, Marshfield, WI 54449, USA

bUniversity of Wisconsin School of Medicine, Madison, WI, USA

${ }^{\mathrm{c} C a r d i o v a s c u l a r}$ Research Institute, Huntington Medical Research Institutes (HMRI), 686 South Fair Oaks Avenue, Pasadena, CA, USA

${ }^{\mathrm{d} C a r d i o v a s c u l a r}$ Division, Department of Medicine, Keck School of Medicine of University of Southern California, Los Angeles, CA, USA

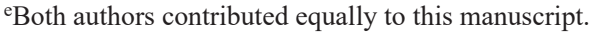

${ }^{f}$ Corresponding Author: Shereif H. Rezkalla, Department of Cardiology \& Cardiovascular Research, Marshfield Clinic Health System, 1000 North Oak Avenue, Marshfield, WI 54449, USA.

Email: rezkalla.shereif@marshfieldclinic.org

doi: https://doi.org/10.14740/cr1290 of some viruses, however, symptoms may linger to the extent the victim is unable to return to a normal lifestyle or gainful employment. Following the severe acute respiratory syndrome (SARS) pandemic of $2003,87 \%$ of patients remained symptomatic with fatigue, weakness, and myalgia that persisted for many months following the infection [2]. Furthermore, it appears that post-infection symptoms were related to the severity of the disease [3]. The coronavirus disease 2019 (COVID-19) infection is no exception to this phenomenon. While many patients had asymptomatic or mild infection, those patients with severe illness continued to have lingering symptoms, although even those with initially mild symptoms can still have "long hauler syndrome", in which symptoms persist beyond 4 weeks [4]. This report will discuss post-COVID symptoms with emphasis on the cardiovascular system.

\section{Post Viral Syndrome}

At the time of this writing (May 2021), the COVID-19 pandemic has resulted in over 160 million individual cases worldwide, with over 3.3 million deaths. In the United States of America, there have been over 33 million cases and 597,484 deaths. While it has been over a year since the pandemic began, the full extent and impact of the post-acute COVID-19 syndrome remains to be fully determined. It is helpful to examine previous coronavirus infections to try to understand the possible challenges we may face today and in the future. Following the severe acute respiratory syndrome coronavirus (SARS-CoV-1) epidemic of 2003, significant numbers of patients who contracted the disease and survived, continued to demonstrate impaired exercise capacity and lung diffusion capacity with up to 15 years of follow-up [5, 6]. After patients recovered from the acute illness of Middle East respiratory syndrome coronavirus (MERS-CoV), 36\% continued to have abnormal chest radiographs and a decrease in lung diffusion capacity [7]. Most of the improvement in the patients' diffusion capacity occurred in the first 2 years after the initial infection. Thus, there is a history of post-viral syndromes even before COVID-19.

Numerous acronyms have been given to the syndrome of post-acute COVID-19 symptoms including long COVID syndrome, long haulers, and post-acute sequelae of SARS-COVID-2 (PASC) syndrome. Going forward in this article, we will refer to the syndrome as PASC.

Follow-up of COVID-19 patients in multiple countries have shown similar findings, with residual symptoms that 


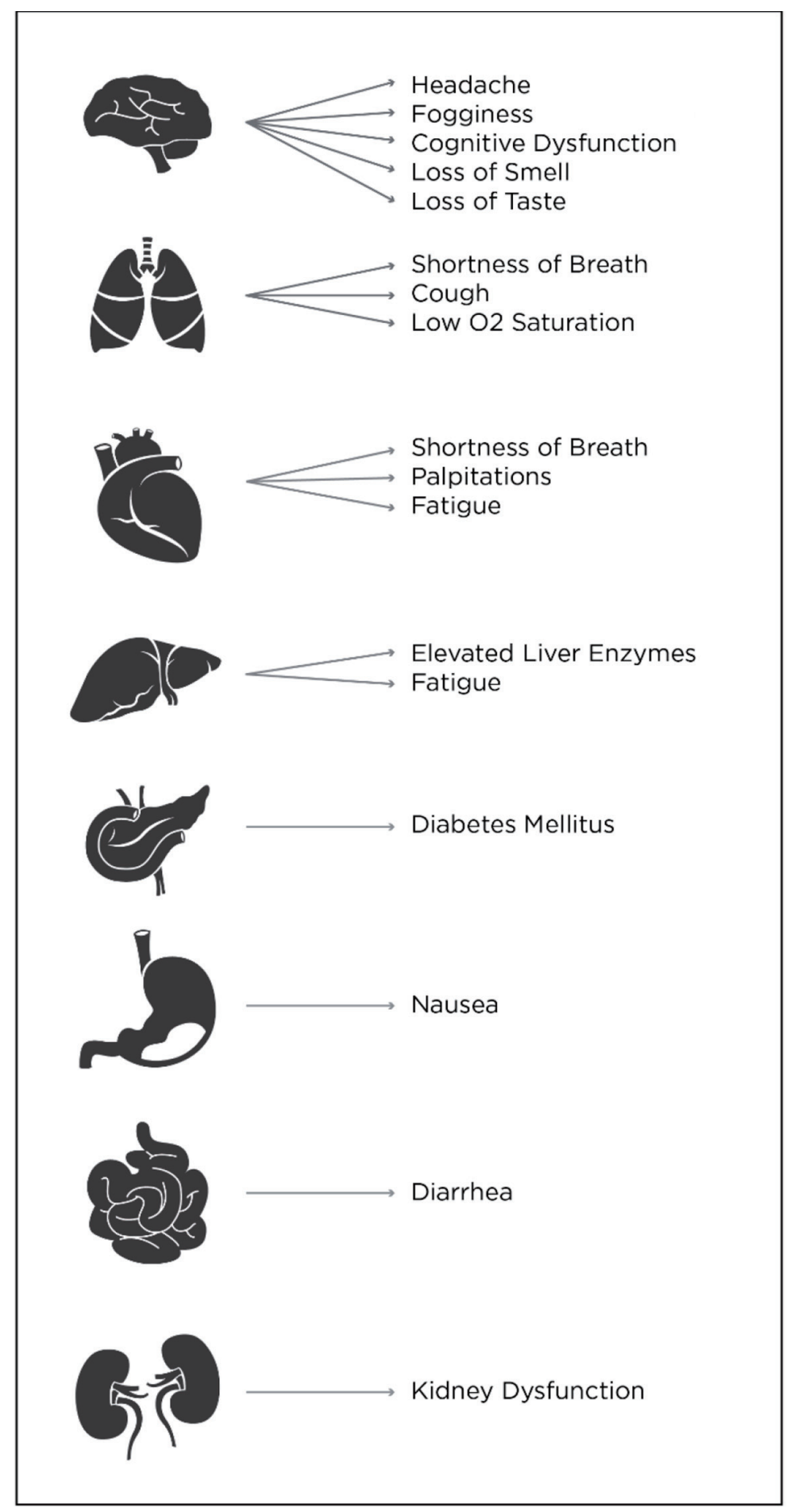

Figure 1. Summary by organ of the most common complaints seen with post-acute sequelae of SARS-COVID-2 (PASC) syndrome.

lasted for many months after resolution of the infection in up to $70 \%$ of the studied cohort $[8,9]$. Patients who experienced PASC syndrome included those with mild as well as severe infection, those who were treated as outpatients, and those who were treated as inpatients. In general, those patients who had more severe acute disease were more likely to experience
PASC syndrome and were more likely to have continued worse symptoms. Up to $30 \%$ of COVID-19 patients may experience PASC syndrome [10]. It is our opinion that the large number of such patients may require organized multi-disciplinary, medical care in the near future, and assurance of appropriate access to such care [11]. Figure 1 summarizes the most common com- 
plaints seen with this syndrome. Following is a discussion of specific organs that may be affected.

\section{Pulmonary System}

Shah et al [12] confirmed that abnormal pulmonary function occurred in $58 \%$ of patients, and abnormal chest computed tomography (CT) images persisted up to 3 months after infection. In postmortem examination of COVID-19 patients, extensive lung fibrosis and inflammation, abnormalities in the liver, and myocarditis were detected. In addition, microangiopathy and fibrosis were detected in more than one patient [13]. It is not surprising that many patients continue to have symptoms of shortness of breath, fatigue, cough, and other symptoms long after the active infection has resolved.

Follow-up of 384 patients who were studied in the United Kingdom for 2 months after contracting COVID-19 showed that $53 \%$ of patients complained of persistent shortness of breath, $34 \%$ continued to have a cough, and about $70 \%$ continued to complain of fatigue [14]. Only $27 \%$ of patients showed improvement on follow-up chest radiography.

In a prospective study of 277 patients recovered from COVID-19 infections, about half continued to have pulmonary symptoms, including shortness of breath and cough within 3 months of the infection. The symptoms ranged from mild to severe, with most in the mild category [15]. Abnormalities in spirometry testing were detected in $9.3 \%$ of patients, and abnormal chest radiography persisted in $18.9 \%$. Some patients required readmission, particularly those with preexisting chronic obstructive pulmonary disease (COPD) and hypertension [16]. While the exact cause of persistent respiratory symptoms is not well defined, it may be related to pulmonary fibrosis [17]. The invasion of mast cells and the development of cytokine storm stimulate fibroblasts and lead to the development of pulmonary fibrosis. In patients with preexisting COPD, the persistent symptoms may be more pronounced and last for a longer postinfection period.

\section{Central Nervous System}

Chronic fatigue syndrome and a variety of psychiatric disorders persisted for years after the SARS pandemic in 2003 [18]. Loss of smell and taste are common symptoms in COVID-19 infections. Occasionally, they linger for 1 or 2 months after infection, and they are likely related to viral effects on the cranial nerves. Occasionally, patients may have myalgias and fatigue for variable amounts of time, similar to myalgic encephalomyelitis/ chronic fatigue syndrome [19]. Both depression and migrainelike headaches have been reported, and patients may report "brain fog" [4]. There is a paucity of data, however, on the incidence or duration of this condition in the post-COVID period.

\section{Gastrointestinal System}

Various gastrointestinal symptoms may manifest during viral infection such as nausea, anorexia, and diarrhea. COVID-19 infection may alter the intestinal flora with depletion of beneficial microorganisms. This may lead to persistent diarrhea in some patients with PASC syndrome. Some other gastrointestinal conditions, such as dyspepsia and irritable bowel syndrome, may be present in patients long after the acute infection has resolved [4]. Additionally, elevated liver enzymes including alanine aminotransferase and aspartate aminotransferase may develop either secondary to the viral infection, hypoxiaischemia reperfusion injury or cytokine storm [20]. The elevated liver enzymes may persist for a few months after infection, particularly if the liver injury is severe [21]. In general, slow recovery occurs, and the liver enzymes eventually normalize.

\section{Miscellaneous}

Other organ systems have been implicated in post-COVID-19 syndrome. When there is severe infection with its associated hypoxemia, diarrhea, and intense cytokine storm, chronic kidney disease may develop. It may persist in the post-infection phase. More recently, new onset diabetes mellitus and worsening of preexisting diabetes have been reported during and following infection $[22,23]$.

\section{Cardiovascular Effects}

During the COVID-19 pandemic, $36 \%$ of patients had elevated cardiac troponin levels with associated electrocardiographic and echocardiographic abnormalities during the acute phase [24]. This results from cardiac effects that vary from myocardial infarction, either ST-elevation infarction or type II infarction, viral myocarditis, or the intense cytokine storm $[25,26]$. Few papers discussed the cardiovascular effects during the PASC syndrome. The cardiovascular findings described in the chronic post-COVID-19 phase have been variable [27]; some reports describe significant cardiovascular effects, while others report only occasional symptoms. During the acute phase of COVID-19 infection, patients may experience symptoms of shortness of breath, chest pain, and palpitation. These symptoms may persist in post-COVID-19 patients up to 6 months after infection, and long after recovery of the viral infection [4]. During the acute phase of viral infection, myocarditis and acute myocardial infarction may occur [28]. Each specific cardiac pathology, such as myocardial infarction or myocarditis, will result in different sequelae from systolic and diastolic cardiac dysfunction, resulting in congestive heart failure to various ventricular and atrial arrhythmias [29]. Utilizing cardiac magnetic resonance imaging (MRI) of 26 patients, myocardial fibrosis and edema were observed in over half of the patients. Cardiac magnetic resonance was obtained about 6 weeks after infection, long after patients were discharged [30]. Endomyocardial biopsy was performed in those patients with abnormal cardiac MRI, revealing lymphocytic infiltration in over $80 \%$ of patients.

In a follow-up, done 60 days after hospital discharge, of 1,250 patients who were discharged from 38 hospitals in Mich- 
igan following recovery from COVID-19 infections, 30\% had persistent symptoms, such as cough, shortness of breath, and fatigue. An additional $9 \%$ of the patients could not return to work, with resultant financial impact [31]. Patients who had long-term cardiac symptoms and persistent abnormal cardiac magnetic resonance imaging (MRI) in the post-COVID period underwent myocardial biopsy. In this study, lymphocytic infiltration also was observed 3 months after infection [32], confirming the report by Huang et al. Other clinical reports confirmed late cardiac symptoms and abnormalities up to 60 days after the active infections had resolved [33, 34]. In a rare prospective study by Sonnweber et al [35], 145 patients with COVID-19 were followed for 100 days; $41 \%$ of patients exhibited symptoms, mainly shortness of breath. While mainly persistent abnormalities were noted in the pulmonary system, cardiac impairments were also noted. These included reduced left ventricular ejection fraction and pulmonary hypertension. Pericardial effusion was only noted in a small number of patients. Rivera-Morales et al [36] described a case of acute myocarditis 2 months following COVID-19 infection in a patient who presented as acute infarction. Coronary angiogram and echocardiography, together with the clinical course, were diagnostic. While it could be simply a sporadic case unrelated to COVID-19, it is intriguing, since there is a group of patients following COVID-19 infection who continue to have elevated high-sensitivity C-reactive protein (hs-CRP). To our knowledge, there are no data available as to the incidence or significance of this elevation, and it could be one phenomenon that requires further research.

Perhaps the most sensitive diagnostic test for such patients is cardiac MRI. In addition to the anatomic and functional assessment of the heart, late gadolinium enhancement, combined with T1 and T2 mapping, provides an idea about the tissue characterization of scar tissue, fibrosis, and edema. In a paper by Kotecha et al [37], 148 patients underwent cardiovascular magnetic resonance at a median of 68 days following infection. Only $10 \%$ had a decrease in left ventricular function, yet 54\% demonstrated late gadolinium enhancement, with myocarditis-like lesions in $26 \%$ of patients. In a report from Germany by Puntmann et al [38], 100 patients were examined using MRI 64 - 92 days post infection. Scans were abnormal in 78 patients, with raised myocardial T1, T2, late gadolinium enhancement, and pericardial enhancement. Endomyocardial biopsy in some patients confirmed active lymphocytic infiltration. The abnormalities were reported in both right and left ventricles [30]. The confirmed presence of myocardial fibrosis may have future implications, since it may trigger cardiac arrhythmias and even sudden cardiac death [26, 39]. In addition to clinical and laboratory evaluation, electrocardiography and echocardiography, MRI is more valuable in diagnosing various residual effects.

Thus, late cardiovascular sequelae of COVID-19 have been reported; however, their exact frequency remains to be determined by larger prospective studies. After resolution of the active infection, patients experience persistent cardiac symptoms such as shortness of breath, chest pain, dizziness, and fatigue. These symptoms may worsen after physical activity. Patients may continue to have abnormal electrocardiogram, echocardiogram, or cardiac magnetic resonance. In this evolving new condition, there is a need for clinical registries and future research to understand the incidence and best management of this syndrome.

\section{Mechanism}

There is no clear single mechanism that may be responsible for PASC syndrome. The one clear finding is that many of the effects of the active phase of the virus linger after resolution of infection. A patient with preexisting COPD may have significant added lung fibrosis with worsening of symptoms and may be even oxygen dependent. Another patient with development of myocarditis during the acute phase may develop congestive heart failure and a variety of atrial and ventricular arrhythmias [40].

There is also clear evidence that markers of the inflammation continue in some patients long after the infective phase [34]. That, as well as mast cell activation observed during both the acute phase of the infection and the post-infection phase [41], may be responsible for some of the symptoms noted in post-COVID-19 patients. An important mechanism that may be implicated in this condition is that the virus that caused COVID-19 is attached to the pericytes and endothelium of the small blood vessels. This mechanism may lead to many adverse effects including vascular endothelial damage, resulting in vascular microthrombosis as well as pericyte damage in the contraction phase [42]. All these effects may result in tissue hypoxemia with various organ system involvements. While these vascular injuries play an integral role in the acute phase of the disease, they may also be responsible for the persistent symptoms in patients with PASC syndrome. They are likely responsible for the new onset diabetes that may develop during the acute viral illness and may persist in some patients during recovery. With this syndrome in its infancy, we anticipate better understanding of the condition with more research in the future.

\section{Management of Post-COVID Syndrome}

In a sample of 100 survivors of the COVID-19 infection, patients were followed for about 9 weeks in the United Kingdom. Symptoms were noted in about $70 \%$ of patients, ranging from fatigue, to shortness of breath, mental fogginess, and a variety of psychologic abnormalities [43]. The involvement of many systems dictates that management of post-COVID syndrome should start with a comprehensive history and physical examination. Further pathways of management will focus on the predominant symptoms. In a patient with pulmonary obstructive lung disease who had significant worsening of shortness of breath or need for home oxygen use, evaluation should begin with chest radiography and pulmonary function testing. Care should be coordinated with a pulmonary specialist. In a patient with marked increase in troponin and with dyspnea or chest discomfort, evaluation should include an electrocardiogram or echocardiogram. If the echocardiogram does not provide a conclusive diagnosis, then cardiac MRI should be considered. Other symptoms, including gastrointestinal, renal, 


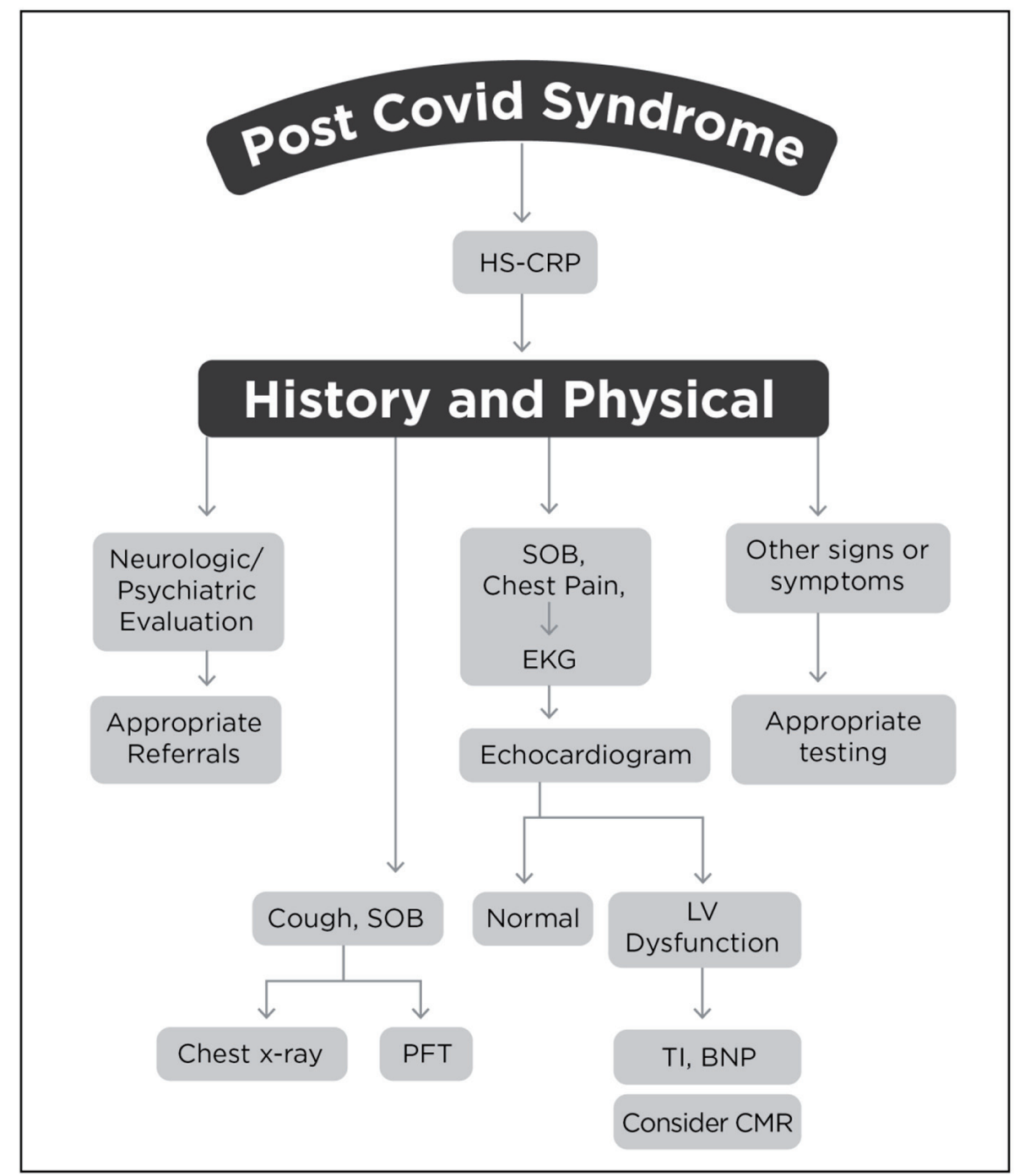

Figure 2. Graphical guide to management of PASC. HS-CRP: high-sensitivity C-reactive protein; SOB: shortness of breath; EKG: electrocardiogram; LV: left ventricle; PFT: pulmonary function test; BNP: brain natriuretic peptide; TI: troponin 1; CMR: cardiac magnetic resonance.

or other organ involvement should be managed accordingly with guidance through the appropriate specialty service. One of the leading challenges is fatigue, with people struggling to return to normal daily life, but they are easily exhausted, have malaise, and tire even with little activity, a condition similar to chronic fatigue syndrome or myalgic encephalomyelitis [44]. Therapy for this condition clearly will require multiple specialties including neurologic and psychiatric collaboration. Most of these patients will require a battery of blood tests to guide management, including a complete blood count, basic metabolic panel, cardiac troponin, brain natriuretic peptide, and liver functions. In addition, a high sensitivity $\mathrm{C}$-reactive protein is essential [45]. In our opinion, continuing evidence of systemic inflammation is the most prominent unknown in this group of patients. Is this the natural effect of the systemic inflammation that occurs during the active infection, that will likely decrease with time, or will the active systemic inflam- mation lead to further organ damage? Only continuing active research studies will answer this enigma [46]. Figure 2 shows a guide to management. We envision the need for organized registries and clinical trials [25] as well as specialized postCOVID-19 clinics to provide answers to these multiple questions [47].

\section{Conclusions}

Along with the initial pandemic of COVID-19 in 2020 - 2021, medical providers may now be facing another new pandemic. Post-COVID syndrome or PASC is an emerging condition that may include the sequelae of the original infection on various body organs and the residual symptoms that may or may not be well defined. Moreover, the continuing systematic inflammation has not been well investigated. In addition, there are very 
little definitive data about the frequency of this syndrome [27]. If the virus lingers in our communities in the future, there is the potential of recurrences of these long-term effects that may increase exponentially [7]. To set appropriate medical policy and management, more understanding of this phenomenon is essential. Appropriate clinical registries, controlled studies, and organized post-COVID clinics are needed to gain better understanding of this syndrome and to provide the best quality care to its victims.

\section{Acknowledgments}

The authors acknowledge Marie Fleisner for editorial assistance with the paper and Kaitlyn Zblewski for creating the figures.

\section{Financial Disclosure}

Dr. Kloner's work was supported in part by the Francis Bacon Foundation and the Pasadena Community Foundation-John and Lucille Crumb Medical Research Endowment to HMRI, and by the Marylou Ingram Endowment to HMRI.

\section{Conflict of Interest}

None to declare.

\section{Author Contributions}

Both Shereif Rezkalla, MD and Robert Kloner, MD did the search, review, critical assessment and writing for this article.

\section{Data Availability}

The authors declare that data supporting the findings of this study are available within the article.

\section{References}

1. Davis DJ. Measurements of the prevalence of viral infections. J Infect Dis. 1976;133(Suppl):A3-5.

2. Moldofsky H, Patcai J. Chronic widespread musculoskeletal pain, fatigue, depression and disordered sleep in chronic post-SARS syndrome; a case-controlled study. BMC Neurol. 2011;11:37.

3. Hickie I, Davenport T, Wakefield D, Vollmer-Conna U, Cameron B, Vernon SD, Reeves WC, et al. Post-infective and chronic fatigue syndromes precipitated by viral and non-viral pathogens: prospective cohort study. BMJ. 2006;333(7568):575.

4. Nalbandian A, Sehgal K, Gupta A, Madhavan MV, McGroder C, Stevens JS, Cook JR, et al. Post-acute COV-
ID-19 syndrome. Nat Med. 2021;27(4):601-615.

5. Hui DS, Joynt GM, Wong KT, Gomersall CD, Li TS, Antonio G, Ko FW, et al. Impact of severe acute respiratory syndrome (SARS) on pulmonary function, functional capacity and quality of life in a cohort of survivors. Thorax. 2005;60(5):401-409.

6. Zhang P, Li J, Liu H, Han N, Ju J, Kou Y, Chen L, et al. Long-term bone and lung consequences associated with hospital-acquired severe acute respiratory syndrome: a 15 -year follow-up from a prospective cohort study. Bone Res. 2020;8:8.

7. Higgins V, Sohaei D, Diamandis EP, Prassas I. COVID-19: from an acute to chronic disease? Potential long-term health consequences. Crit Rev Clin Lab Sci. 2021;58(5):297-310.

8. Goertz YMJ, Van Herck M, Delbressine JM, Vaes AW, Meys R, Machado FVC, Houben-Wilke S, et al. Persistent symptoms 3 months after a SARS-CoV-2 infection: the post-COVID-19 syndrome? ERJ Open Res. 2020;6(4):00542-2020.

9. Carfi A, Bernabei R, Landi F, Gemelli Against C-P-ACSG. Persistent symptoms in patients after acute COVID-19. JAMA. 2020;324(6):603-605.

10. Logue JK, Franko NM, McCulloch DJ, McDonald D, Magedson A, Wolf CR, Chu HY. Sequelae in adults at 6 months after COVID-19 infection. JAMA Netw Open. 2021;4(2):e210830.

11. Ladds E, Rushforth A, Wieringa S, Taylor S, Rayner C, Husain L, Greenhalgh T. Persistent symptoms after Covid-19: qualitative study of 114 "long Covid" patients and draft quality principles for services. BMC Health Serv Res. 2020;20(1):1144.

12. Shah AS, Wong AW, Hague CJ, Murphy DT, Johnston JC, Ryerson CJ, Carlsten C. A prospective study of 12-week respiratory outcomes in COVID-19-related hospitalisations. Thorax. 2021;76(4):402-404.

13. Cennimo DJ, Bergman SJ, Olsen KM. Coronavirus disease 2019 (COVID-19) clinical presentation. Medscape. Updated April 2, 2021. Available at: https://emedicine. medscape.com/article/2500114-clinical.

14. Mandal S, Barnett J, Brill SE, Brown JS, Denneny EK, Hare SS, Heightman M, et al. 'Long-COVID': a crosssectional study of persisting symptoms, biomarker and imaging abnormalities following hospitalisation for COVID-19. Thorax. 2021;76(4):396-398.

15. Moreno-Perez O, Merino E, Leon-Ramirez JM, Andres M, Ramos JM, Arenas-Jimenez J, Asensio S, et al. Postacute COVID-19 syndrome. Incidence and risk factors: A Mediterranean cohort study. J Infect. 2021;82(3):378383.

16. Somani SS, Richter F, Fuster V, De Freitas JK, Naik N, Sigel K, Mount Sinai CIC, et al. Characterization of patients who return to hospital following discharge from hospitalization for COVID-19. J Gen Intern Med. 2020;35(10):2838-2844.

17. Kazama I. Stabilizing mast cells by commonly used drugs: a novel therapeutic target to relieve post-COVID syndrome? Drug Discov Ther. 2020;14(5):259-261.

18. Lam MH, Wing YK, Yu MW, Leung CM, Ma RC, Kong 
AP, So WY, et al. Mental morbidities and chronic fatigue in severe acute respiratory syndrome survivors: long-term follow-up. Arch Intern Med. 2009;169(22):2142-2147.

19. Nath A. Long-Haul COVID. Neurology. 2020;95(13):559560.

20. Zhong $\mathrm{P}, \mathrm{Xu}$ J, Yang D, Shen Y, Wang L, Feng Y, Du C, et al. COVID-19-associated gastrointestinal and liver injury: clinical features and potential mechanisms. Signal Transduct Target Ther. 2020;5(1):256.

21. McDermott CV, Cox EJ, Scanlan JM, Alicic RZ. COVID-19 and gastrointestinal tract symptoms: recognition, containment, and learning from the past. Mayo Clin Proc. 2020;95(11):2320-2324.

22. Carbajal E. COVID-19 might be triggering new-onset diabetes, researchers say. Hospital Review. February 21, 2021. Available at: https://www.beckershospitalreview. com/patient-safety-outcomes/covid-19-might-be-triggering-new-onset-diabetes-researchers-say.html.

23. Rubino F, Amiel SA, Zimmet P, Alberti G, Bornstein $\mathrm{S}$, Eckel RH, Mingrone G, et al. New-onset diabetes in COVID-19. N Engl J Med. 2020;383(8):789-790.

24. Rezkalla SH, Kloner RA. Viral myocarditis: 1917-2020: From the Influenza A to the COVID-19 pandemics. Trends Cardiovasc Med. 2021;31(3):163-169.

25. Mitrani RD, Dabas N, Goldberger JJ. COVID-19 cardiac injury: Implications for long-term surveillance and outcomes in survivors. Heart Rhythm. 2020;17(11):19841990 .

26. Umbrajkar S, Stankowski RV, Rezkalla S, Kloner RA. Cardiovascular health and disease in the context of COVID-19. Cardiol Res. 2021;12(2):67-79.

27. Maleszewski JJ, Young PM, Ackerman MJ, Halushka MK. Urgent need for studies of the late effects of SARS-CoV-2 on the cardiovascular system. Circulation. 2021;143(13):1271-1273.

28. Pirzada A, Mokhtar AT, Moeller AD. COVID-19 and myocarditis: what do we know so far? CJC Open. 2020;2(4):278-285.

29. Yancy CW, Fonarow GC. Coronavirus disease 2019 (COVID-19) and the heart-is heart failure the next chapter? JAMA Cardiol. 2020;5(11):1216-1217.

30. Huang L, Zhao P, Tang D, Zhu T, Han R, Zhan C, Liu $\mathrm{W}$, et al. Cardiac involvement in patients recovered from COVID-2019 identified using magnetic resonance imaging. JACC Cardiovasc Imaging. 2020;13(11):2330-2339.

31. Chopra V, Flanders SA, O'Malley M, Malani AN, Prescott HC. Sixty-day outcomes among patients hospitalized with COVID-19. Ann Intern Med. 2021;174(4):576-578.

32. Becker RC. Anticipating the long-term cardiovascular effects of COVID-19. J Thromb Thrombolysis. 2020;50(3):512-524.

33. Shchendrygina A, Nagel E, Puntmann VO, Valbuena-Lopez S. COVID-19 myocarditis and prospective heart failure burden. Expert Rev Cardiovasc Ther. 2021;19(1):5-14.
34. Doykov I, Hallqvist J, Gilmour KC, Grandjean L, Mills K, Heywood WE. 'The long tail of Covid-19' - The detection of a prolonged inflammatory response after a SARS$\mathrm{CoV}-2$ infection in asymptomatic and mildly affected patients. F1000Res. 2020;9:1349.

35. Sonnweber T, Sahanic S, Pizzini A, Luger A, Schwabl C, Sonnweber B, Kurz K, et al. Cardiopulmonary recovery after COVID-19: an observational prospective multicentre trial. Eur Respir J. 2021;57(4):2003481.

36. Rivera-Morales MD, Pell R, Rubero J, Ganti L. Acute myopericarditis in the post COVID-19 recovery phase. Cureus. 2020;12(10):e11247.

37. Kotecha T, Knight DS, Razvi Y, Kumar K, Vimalesvaran K, Thornton G, Patel R, et al. Patterns of myocardial injury in recovered troponin-positive COVID-19 patients assessed by cardiovascular magnetic resonance. Eur Heart J. 2021;42(19):1866-1878.

38. Puntmann VO, Carerj ML, Wieters I, Fahim M, Arendt C, Hoffmann J, Shchendrygina A, et al. Outcomes of cardiovascular magnetic resonance imaging in patients recently recovered from coronavirus disease 2019 (COVID-19). JAMA Cardiol. 2020;5(11):1265-1273.

39. Junttila MJ, Hookana E, Kaikkonen KS, Kortelainen ML, Myerburg RJ, Huikuri HV. Temporal trends in the clinical and pathological characteristics of victims of sudden cardiac death in the absence of previously identified heart disease. Circ Arrhythm Electrophysiol. 2016;9(6):e003723.

40. Coromilas EJ, Kochav S, Goldenthal I, Biviano A, Garan $\mathrm{H}$, Goldbarg S, Kim JH, et al. Worldwide survey of COVID-19-associated arrhythmias. Circ Arrhythm Electrophysiol. 2021;14(3):e009458.

41. Afrin LB, Weinstock LB, Molderings GJ. Covid-19 hyperinflammation and post-Covid-19 illness may be rooted in mast cell activation syndrome. Int $\mathrm{J}$ Infect Dis. 2020;100:327-332.

42. Ostergaard L. SARS CoV-2 related microvascular damage and symptoms during and after COVID-19: Consequences of capillary transit-time changes, tissue hypoxia and inflammation. Physiol Rep. 2021;9(3):e14726.

43. Halpin SJ, McIvor C, Whyatt G, Adams A, Harvey O, McLean L, Walshaw C, et al. Postdischarge symptoms and rehabilitation needs in survivors of COVID-19 infection: A cross-sectional evaluation. J Med Virol. 2021;93(2):1013-1022.

44. Marshall M. The lasting misery of coronavirus long-haulers. Nature. 2020;585(7825):339-341.

45. Greenhalgh T, Knight M, A'Court C, Buxton M, Husain L. Management of post-acute covid-19 in primary care. BMJ. 2020;370:m3026.

46. Mahase E. Covid-19: What do we know about "long covid"? BMJ. 2020;370:m2815.

47. Rovere Querini P, De Lorenzo R, Conte C, Brioni E, Lanzani C, Yacoub MR, Chionna R, et al. Post-COVID-19 follow-up clinic: depicting chronicity of a new disease. Acta Biomed. 2020;91(9-S):22-28. 